\title{
Correction to: Diagnosis and Treatment of the Occipito-Atlantoaxial Complex and Subaxial Cervical Spine in Rheumatoid Diseases
}

Marcus Richter

\section{Correction to: Chapter 44 in: B. Meyer, M. Rauschmann (eds.), Spine Surgery,} https://doi.org/10.1007/978-3-319-98875-7_44

This chapter has been revised to correct the author name to 'Marcus Richter'. 